\title{
Polarized Raman Confocal Microscopy of Single Gallium Nitride Nanowires
}

Peter J. Pauzauskie, ${ }^{\dagger}$ David Talaga, ${ }^{\ddagger}$ Kwanyong Seo, ${ }^{\dagger} \S$ Peidong Yang ${ }^{\dagger} *$ and François Lagugné-Labarthet ${ }^{\ddagger} * *$

${ }^{\dagger}$ Department of Chemistry, University of California, Berkeley, California 94720, USA and Materials Science Division, Lawrence Berkeley National Laboratory, Berkeley, California, 94720, USA,

${ }^{\ddagger}$ Laboratoire de Physico-Chimie Moléculaire, UMR 5803 CNRS, Université Bordeaux 1, 351 cours de la Libération, 33405 Talence cedex, France.

\section{Supporting information :}

1. Experimental Details

2. References

$\S$ Current address: Department of Chemistry, KAIST, Daejeon 305-701, Republic of Korea

*Corresponding authors: p_yang@uclink.berkeley.edu and f.lagugne@lpcm.u-bordeaux1.fr 


\section{Experimental Details}

Materials Synthesis. Gallium nitride nanowires were synthesized in a horizontal hot-wall furnace (Lindberg Blue) housing a 1" diameter outer quartz flow tube ${ }^{1}$. Nanowires were grown with a chemical vapour transport process in which liquid-metallic gallium was used for a Ga precursor, while mass flow controllers delivered ammonia and hydrogen gases at 15 and $30 \mathrm{sccm}$, respectively. Approximately $20 \mathrm{mg}$ of gallium was positioned on a quartz or alumina substrate and placed $1 \mathrm{~cm}$ upstream from a single-crystal substrate $(\mathrm{MgO}(111), \gamma-$ $\mathrm{LiAlO}_{2}(100)$, or $\mathrm{c}-\mathrm{Al}_{2} \mathrm{O}_{3}$ ) that had been coated with $\sim 2 \mathrm{~nm}$ of nickel via thermal evaporation. Particular substrates were chosen to select the desired nanowire growth direction ${ }^{2}$. The furnace was ramped to $900^{\circ} \mathrm{C}$ in approximately 20 minutes, and the reaction was allowed to take place anywhere from 30 min to 12 hours.

Experimental Methods. A single [001] nanowire with a wurtzite-like hexagonal cross section (confirmed by transmission electron microscopy) was first cut and positioned on a microscope glass slide (Figure 1a). A bent shape was purposely induced using a nanomanipulator and atomic force microscopy (AFM) reveals a diameter of about $170 \mathrm{~nm}$ and a length of $41 \mu \mathrm{m}$. Our Raman instrument is based on an inverted microscope (Olympus IX 71) combined with a Labram HR800 (Jobin-Yvon, Horiba group) spectrometer. The polarized Raman imaging of the nanowire were performed with a 600 grooves/mm grating with a resolution $4 \mathrm{~cm}^{-1}$. The polarized spectra recorded locally over a longer time (no imaging) were recorded with a 1800 grooves $/ \mathrm{mm}$ grating with a resolution of about $0.5 \mathrm{~cm}^{-1}$. The sample is scanned with a piezoelectric stage (PI 540.3 CL) with an intrinsic accuracy of about $1 \mathrm{~nm}$ in the lateral directions. The light beam input excitation wavelength was fixed at $\lambda=514.5 \mathrm{~nm}$ from an $\mathrm{Ar}^{+}$ion laser and the input polarization was selected with a half-waveplate. Focussing on the sample was done with a 100X objective (Olympus MPL 100X-NA=0.90) 
and the Raman signal was collected under the backscattering geometry. A polarizer serves as an analyzer just in front of the entrance slit of the spectrometer. The laser intensity was fixed to $10 \mathrm{~mW}$ at the focal point and the depolarization ratios of our instrument were checked with a reference sample of liquid $\mathrm{CCl}_{4}$. The pinhole of the confocal microscope was closed at about 140 microns, which corresponded to a focal volume of about 1 micron cube, limiting significantly the background signal of the glass substrate. The detector was a liquid-nitrogen cooled CCD camera and acquisition time was fixed to 1s per spectrum in the mapping mode while an acquisition time of 20 s per spectrum was used to record most spectra shown in the present work.
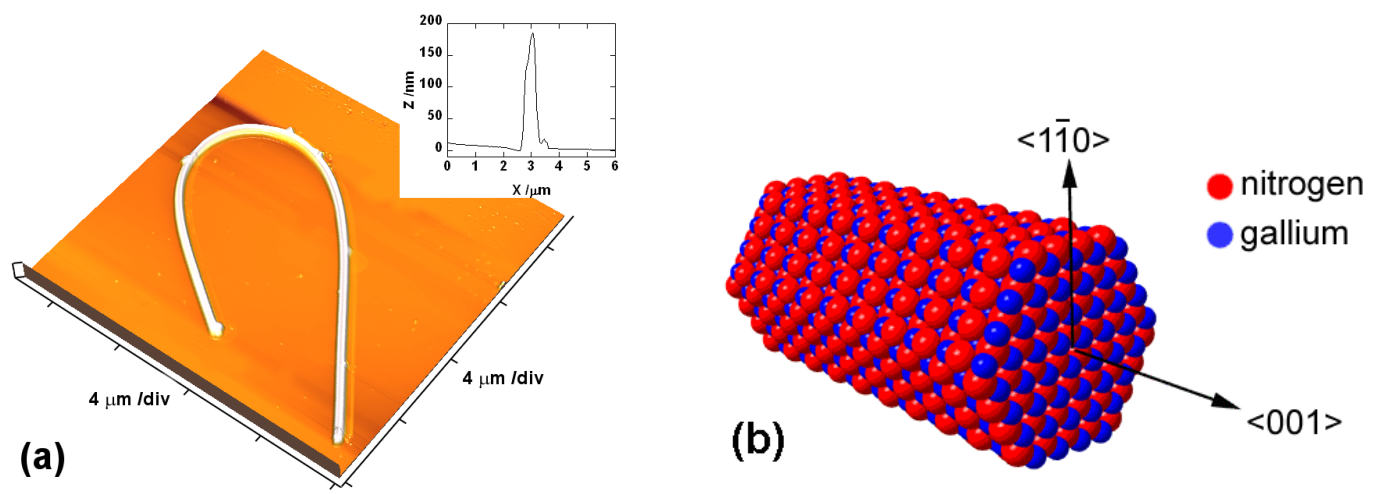

Figure 1: Cross section and atomic force microscopy topographical image (a) of the [001] (b) Lattice rendering (Crystal Impact, GbR) wurtzite GaN nanowire deposited on a glass substrate. Arrows correspond to orthogonal crystallographic growth directions of wires used in this work.

Raman Imaging. Mapping of the nanowire was performed by recording step-spectra every $200 \mathrm{~nm}$ with an integration time of 1s. Due to the highly focussed laser beam, a short acquisition time was possible, reducing the occurrence of any possible thermal effects. A nonresonant 514.5 $\mathrm{Ar}^{+}$laser was used in this work to avoid these complications. Furthermore, no spectral shifts were observed with longer collection times, suggesting that laser heating is not affecting the crystal lattice. Images are shown in Figure 2 before and after smoothing for the 
$Y(Z Z) \bar{Y}$ polarization configuration and represent the variation of the integrated Raman signal over the [509-552] $\mathrm{cm}^{-1}$ spectral range. Before image interpolation, each pixel in Figure 2 corresponds to a surface of $(200 \times 200) \mathrm{nm}^{2}$. It is noteworthy that at the focal point, the laser beam has a diameter of about 1 micron which is 5 times larger that the recording step used for the mapping of the nanowire. The corresponding width of the integrated Raman image of the nanowire is then about 4-5 pixels, in agreement with a nanowire diameter less than $200 \mathrm{~nm}$. The spectra in figure 3 correspond to different portions of the nanowire and to the background signal from the glass substrate.
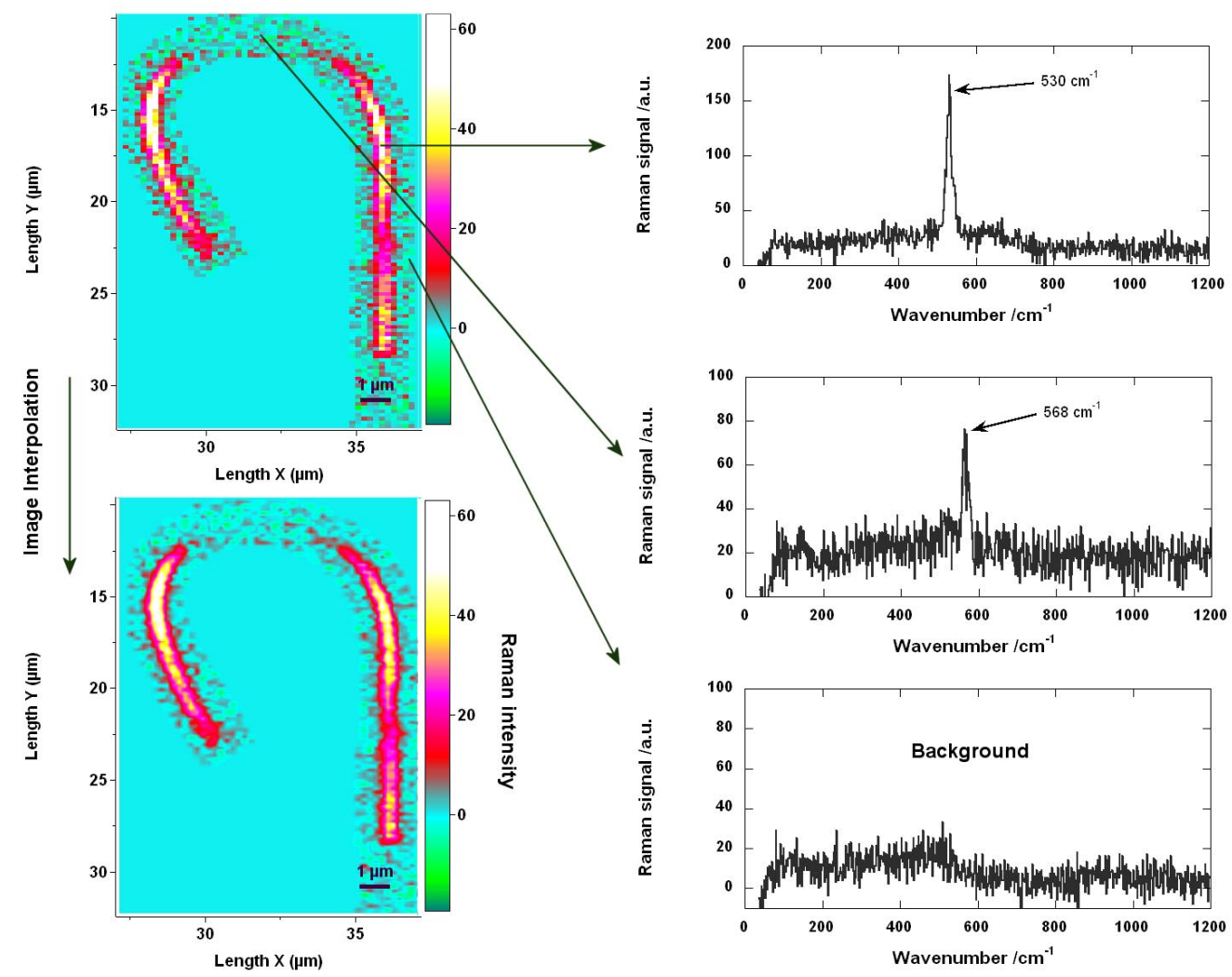

Figure 2. Raman mapping of the nanowire in the $Y(Z Z) \bar{Y}$ polarization configuration before and after image interpolation. Each pixel corresponds to a spectrum recorded on a surface of (200x200) $\mathrm{nm}^{2}$ and integrated over the [509-552] $\mathrm{cm}^{-1}$ spectral range. The spectra on the left are taken in the parts of the images indicated by the arrows (acquisition time 1s per spectra). 


\section{Raman spectra of $[1 \overline{1} 0]$ nanowires.}

To clearly identify the spectral position of the $559 \mathrm{~cm}^{-1}$ and $568 \mathrm{~cm}^{-1}$ modes, the Raman spectra of $[1 \overline{1} 0] \mathrm{GaN}$ nanowires were both recorded at low and high spectral resolution under the microscope. However, as shown in Figure 3, the spectra recorded under the $Y(X X+X Z) \bar{Y}$ polarization combination exhibits a stronger background when a higher spectral resolution is used while it is strongly reduced at lower spectral resolution. The use of a high density grating (1800 grooves/mm) for higher resolution measurements and different confocal conditions (confocal hole more open to compensate the weaker throughput of the spectrometer) are in part responsible for a larger background coming from the glass substrate onto which the nanowire was deposited.

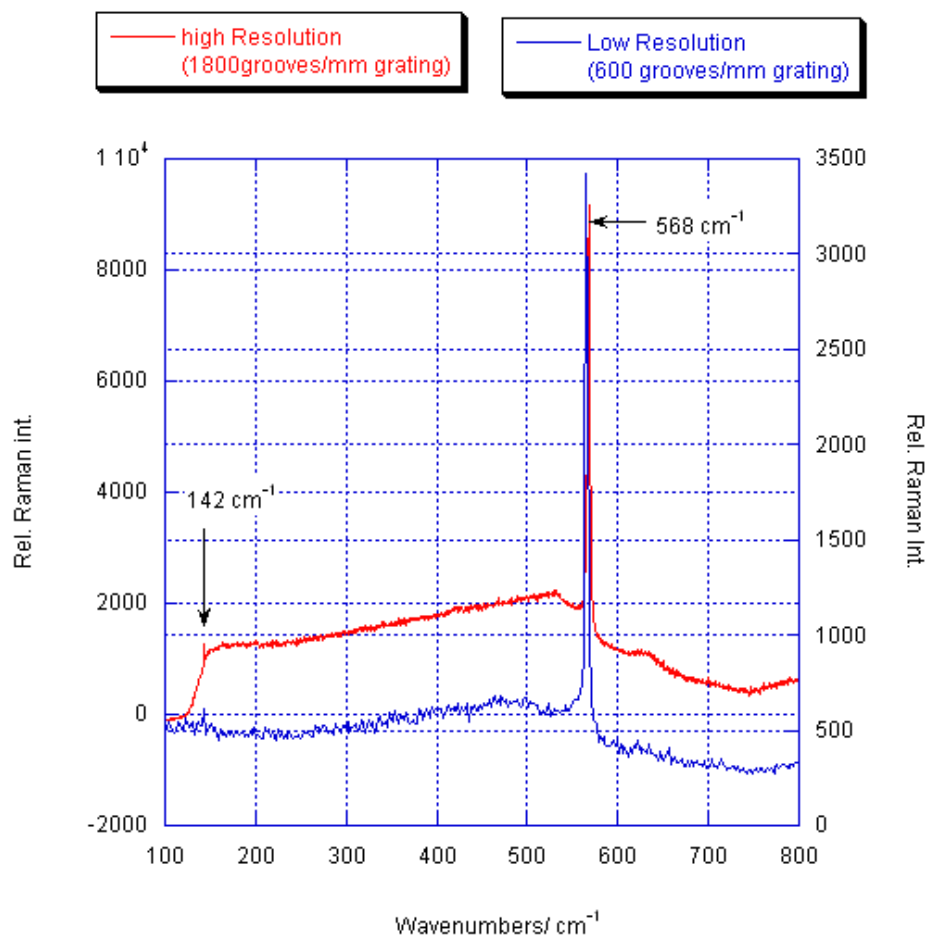

Figure 3. Raman spectra recorded with different spectral resolution $\left(1.4 \mathrm{~cm}^{-1}\right.$ for the 1800 grooves $/ \mathrm{mm}$ and $4 \mathrm{~cm}^{-1}$ for the 600 grooves $/ \mathrm{mm}$ ) of a single [1 10 ] nanowire in the $Y(X X+X Z) \bar{Y}$ polarization combination. 


\section{References}

(1) Johnson, J. C.; Choi, H.-J.; Knutsen, K. P.; Schaller, R. D.; Yang, P.; Saykally, R. J. Nature Materials 2002, 1, 106.

(2) Kuykendall, T.; Pauzauskie, P.; Zhang, Y.; Goldberger, J.; Sirbully, D.;

Denlinger, J.; Yang, P. Nature Materials 2004, 3, 524. 